\section{Word class and serial position in the immediate recall of sentences}

\author{
ALEXANDER J. WEARING* $†$ \\ Yale University, New Haven, Conn. 06510
}

A divided-attention task was used to study the immediate recall of sentences and to determine whether or not nouns were recalled better than modifiers, verbs were recalled better than nouns, and a normal skewed U-shaped serial position curve could be detected. The answer to all three questions was a qualified yes.

Conventional wisdom has it that when a sentence is learned, the core meaning (which term usually involves the agent (logical subject), object, and verb] is stored, while modifiers such as adjectives and adverbs are quickly forgotten (Mandler \& Mandler, 1964; Martin, Roberts, \& Collins, 1968; Martin \& Walter, 1969). Belief in the storage of the core meaning usually presumes that normal serial position effects (Crowder, 1969) are overridden and that those terms comprising the core meaning are all stored equally well.

However, neither the role of serial position nor the possibility that the core meaning may not be equally strongly carried by the subject, object, and rerb has been thoroughly investigated. Fodor, Garrett, \& Bever (1968) present evidence for a central role for the verb in sentence processing, and Gladney \& Krulee (1967) found that changes in the verb caused more disruption in sentence recognition and recall than did changes in the subject or the object of the sentence. Healy \& Miller (1970) showed that when sentences are sorted on the basis of similarity of meaning, the verb is a much better predictor of sorting decisions than either the subject or the object.

On the other hand, Wearing (1969a, b, 1970) found that in both immediate $(30-\mathrm{min})$ and $48-\mathrm{h}$ recall, the subject and object were both recognized more easily and were more effective recall cues than the main verb of the sentence. Martin, Roberts, \& Collins (1968) found that for four different kinds of sentences, at 0 and $10 \mathrm{sec}$ after presentation, the verb was recalled worst on five of the eight occasions but never was recalled best. For Martin \& Walter (1969), with

*This study was supported by NTMH Grant MH-17706-01. The author is grateful to $M$. B. Parker and Rosemary Wearing for recording the sentences and digits, to Bruce Parker for collecting the data, and to Bruce Parker and Rosemary Wearing for assistance in its analysis.

+Present address is: Department of Psychology, University of Melbourne, Parkville, Victoria 3052, Australia. three kinds of sentences at 0 - and 10 -sec recall intervals, the verb was recalled worst on two of the six occasions and never recalled best. A careful examination of Mandler \& Mandler's (1964) figures also suggests that recall of the verb was inferior to that of the nouns, at least on the early trials.

But perhaps the most notable unstated result of these last three experiments is that far from being unimportant, the normal serial position effects are quite strongly manifested. In the Martin et al experiments, the single best predictor of recall is serial position, and in the Mandler \& Mandler (1964) experiments, serial position effects are also strongly pronounced.

The purpose of the present experiment was to answer the questions of whether or not form class and serial position effects can be reliably observed in sentence processing. The most frequently used procedure for studying this in short-term memory has been the procedure due to Peterson \& Peterson (1959), but in the present case it seemed desirable to use a technique which permitted immediate recall, yielded a reasonable error rate, and, in addition, simulated normal speech processing conditions to the extent that attention was only partly directed to the speaker. A procedure that meets these requirements is a divided-attention task of the kind described by Wearing \& Crowder (1971) in which the listener is required to attend to some other competing input as well as the sentence itself. SUBJECTS

The Ss were 26 undergraduate volunteers from Yale University.

\section{MATERIALS}

Thirty experimental sentences were embedded in a list of 38 sentences. All sentences were 11 words in length. All the experimental sentences had the same grammatical structure, being of the form: Preposition + Determiner + Adjective + Noun + Determiner + Adjective + Noun + Verb + Determiner + Adjective + Noun. The semantic content of the sentences varied widely, and no sentences had any content words in common. The main purpose of the buffer sentences (also 11 words in length) were to absorb warmup effects at the beginning of the task and to provide some variation in the course of the list. The experimental sequence was the same for all Ss.

The sentences and the digits were recorded simultaneously with a stereo tape recorder, the sentences being spoken by a male and the digits by a female. Normal speech rhythms were used, and the location of the 10 digits relative to the 11 words is shown in Fig. 1.

\section{PROCEDURE}

The Ss, who were tested individually, were seated in front of a two-speaker stereo system and were told that they would hear a sentence from one of the speakers at the same time as they heard a set of digits from the other and that after presentation they would have to recall both sets of materials in order. Ss were assigned alternately to one of two experimental groups. They were told that they must first recall the sentence and then the digits, or vice versa. In both cases, they were instructed to focus their attention equally on both inputs. Balancing order of recall seemed a prudent control in the light of Epstein's (1969) finding that a procedure similar to the present one yielded results that were sensitive to the order of recall. Each group contained $13 \mathrm{Ss}$, and each $\mathrm{S}$ received all of the sentences in the same order. RESULTS

The proportion of digits correctly recalled in each serial position is

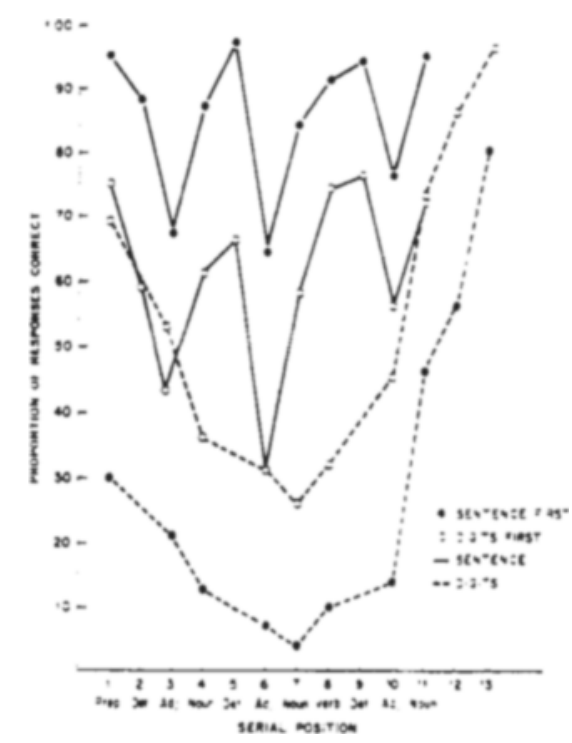

Fig. 1. Proportion of words and digits correct as a function of order of recall and serial position. 
Table 1

Correlations (With Regard to Correctness of Recall) Between Successive Words in the Sentences for the Digits Recalled First and Sentences Recalled First Groups Separately

Form Class

\begin{tabular}{lcccccccccc} 
Preposition & Det. & Adj. & Noun & Det. & Adj. & Noun & Verb & Det. & Adi. Noun \\
\hline Sentence First & .20 & .50 & .29 & .21 & .24 & .27 & .10 & .22 & .07 & .35 \\
Digits First & .58 & .51 & .52 & .38 & .39 & .26 & .14 & .50 & .42 & .45
\end{tabular}

shown in Fig. 1. Recall of digits was better when the digits were recalled first, $F(1,24)=70.34, p<.01$, and the overall effect of serial position was a ls o highly signific an t $[F(9,216)=80.21, p<.01]$.

The corresponding recall curves for the sentences are also shown in Fig. 1. Sentences were recalled better if they were recalled first, $F(1,24)=27.80$, $p<.01$. There were no significant interactions between sentences and digits at recall.

The presence of a serial position effect and the relative goodness of recall of the nouns, on the one hand, and their modifiers, on the other, were tested with orthogonal planned comparisons. From Fig. 1 it can be seen that there were three adjective-noun pairs in each sentence. Overall, the recall of nouns was superior to that of adjectives, $F(1,264)=9.10, p<.01$. A skewed U-shaped function rather than a straight line was found to link the three successive adjective-noun pairs in that recall of the seccind adjective-noun pair was inferior to both the first and the third pair, $F(1,264)=5.36, p<.025$. It may be noted that such a U-shaped function is characteristic of lists of unrelated items.

Because of this evidence of a serial position effect, it becomes somewhat difficult to answer the question about whether the verb or the nouns were recalled better. However, when a local comparison was made between the verb and the immediately preceding word (which is a noun), it was found that the recall of the verb was superior, $t(24)=3.06, p<.01$.

There is a small, but significant, trade-off between the digits and the corresponding words in the sentence. The median correlation between the 16 word-digit pairs which were temporarily adjacent at presentation (with regard to correctness of recall) was -0.03 , the range was from -0.20 to 0.06 , and, since 13 of the 16 correlations were negative, it is apparent that a digit tended to be remembered at the expense of a word (or vice versa) when they arrived simultaneously.

Besides the correlation between recall scores of adjacent words and digits, it is of interest to consider the correlation between the recall performances of the adjacent words, that is, the extent to which recall of one particular word in a sentence is predictive of the immediately following word.

The correlation between the recall of successive words in the sentence is shown in Table 1. Although the correlations are attenuated by a ceiling effect when the sentences are recalled first, it can be seen that the sentence chunks into two parts, corresponding to the traditional distinction between the subject and predicate.

The present results both demonstrate the usefulness of the divided-attention technique and also confirm previous work in finding that the nouns and verb were recalled better than the modifiers. However, the recall of any particular content word depended not only on whether or not it was a modifier, but also on its position in the sentence and whether or not it was a noun or a verb. The skewed U-function characterisitc of the recall of unrelated items (Crowder, 1969) emerged in these results (when word class was controlled), even though it was modified by the linguistic relationships in the word strings. (For example, all adjectives were recalled better than all nouns regardless of their positions in the sentence.) Apart from the fact that the interpretation of word-class studies must now apparently take into account serial position effects, it is worth noting that natural language does, in fact, behave as if the ends of sentences were more important than the middle; for example, the effect of the passive transformation is to give special emphasis to the logical object of a sentence by shifting it to the first serial position.

Although the divided attention technique proved useful in the present experiment, it should be noted that Wearing \& Crowder's (1971) finding that the pattern of digit recall reflected the linguistic structure of the sentence was not replicated. The serial position curve for digits is apparently independent of sentence structure. Since one of Wearing and Crowder's intentions was to devise a technique that would be sensitive to variations in linguistic structure, the present finding places a severe qualification upon their conclusions.

\section{DISCUSSION}

One feature of the findings which may seem a trifle unexpected is the high level of recall for function words. The reason for this is artifactual and results from the standardized sentence frame that was employed.

In five out of six cases, the verb was recalled better than were the nouns (see Fig. 1), and the exception may be a special case because it was the final word in the words recalled in the first condition and so may have been read directly out of primary memory. When compared with the immediately preceding noun, the recall of the verb is superior, a result which confirms those of Gladney \& Krulee (1967) and Fodor et al (1968) [Martin et al (1968) and Martin \& Walter (1969) are difficult to weigh in this matter because of the serial position effects in their data ], but not those of Wearing $(1969 a, b, 1970)$. If it is actually the case that the verb is recalled well from short-term memory but not from long-term memory, then we have raised something of a problem for conventional theories of memory for sentences, which are mainly of the core meaning kind. The possibility that those aspects of a sentence which are important for its understanding may be less important for storage had been considered by Bregman \& Strasberg (1968), who have distinguished empirically between what they call the transmission code and the semantic message. The present results provide some support for their theoretical distinction.

Although opinion is unanimous that modifiers are more poorly remembered than are other content words, the presumed fact is a little puzzling, since it seems reasonable to suppose that there should be occasions when such a finding does not hold. Consider, for example, the sentence, The good man strode toward the helpless child, and a contrasting sentence that differs only in one word, The evil man strode toward the child. A single modifier crucially affects the meaning of the whole sentence, and in order to remember the meaning of the sentence, one would surely have to remember the adjective which modifies man.

The possibility (which received some support in this study) that sentences are remembered in chunks that correspond to grammatical classes indicates that when the other above findings are also considered, the immediate recall of words in sentences is complexly determined by grammatical word class and serial position, as well as the clausal structure of the sentence.

\section{REFERENCES}

BREGMAN, A. S., \& STRASBERG, R. 
Memory for the syntactic form of sentences. Journal of Verbal Learning \& Verbal Behavior, 1968, 7, 396-403.

CROWDER, R. G. Behavioral strategies in immediate memory. Journal of Verbal Learning \& Verbal Behavior, 1969,8 , 524-528.

EPSTEIN, W. Recall of word lists following learning of sentences and of anomalous and random strings. Journal of Verbal Learning \& Verbal Behavior, 1969, 8, 20-25.

FODOR, J. A., \& GARRETT, M. Some syntactic determinants of sentential complexity, II. Verb structure. Perception \& Psychophysics, 1968, 3, $453-461$.

GLADNEY, T. A., \& KRULEE, G. K. The influence of syntactic errors on sentence recognition. Journal of Verbal Learning \& Verbal Behavior, 1967, 6, 692-698.

HEALY, A, F \& MILLER, G, A. The verb as the main determinant of sentence meaning. Psy chonomic Science, 1970. 20, 372.

MANDLER, G., \& MANDLER, J. M. Serial position effects in sentences. Journal of Verbal Learning \& Verbal Behavior. 1964, 3, 195-202.

M ARTIN, E., ROBERTS, K. H., \& COLLINS, A. M. Short-term memory for sentences. Journal of Verbal Learning \& Verbal Behavior, 1968, 7, 560-566.

MARTIN, E., \& WALKER, D. A. Subject uncertainty and word-class effects in short-term memory for sentences. Journa of Experimental Psychology, 1969, 80,
47-51.

PETERSON, L. R., \& PETERSON, M. J. Short-term retention of individual verbal items. Journal of Experimental Psychology, 1959, 58, 193-198.

WEARING, $A$. What is stored when a sentence is learned? Paper read to the Western Psychological Association, 1969 .

WEARING. A. J. Sentence storage and retrieval. Paper read to the Psychonomic Society, $1969 \mathrm{~b}$.

WEARING, A. J. The storage of complex sentences. Journal of Verbal Learning \& Verbal Behavior, 1970, 9, 21-28.

WEARING, A. J., \& CROWDER, R. G. Dividing attention to study sentence acquisition. Journal of Verbal Learning \& Verbal Behavior, 1971, 10, 254-261. 